\title{
Characterization of Langmuir and Langmuir-Blodgett films of a thiomacrocyclic ionophore by surface pressure and AFM
}

J. Torrent-Burgués ${ }^{1(*)}$, M. Pla ${ }^{2}$, Ll. Escriche ${ }^{3}$ J. Casabó ${ }^{3}$, A. Errachid ${ }^{2}$, F. Sanz ${ }^{2,4}$

1 Department of Chemical Engineering, Universitat Politècnica de Catalunya (UPC), C/ Colom 1, 08222 Terrassa (Barcelona), Spain, Tlf. 34 937398043, Fax 34 937398225, e-mail: juan.torrent@upc.edu

2 Laboratory of NanoBioengineering, CBEN-CREBEC, PCB, C/ Josep Samitier 1-5, 08028 Barcelona, Spain

3 Unit of Inorganic Chemistry, Department of Chemistry, Universitat Autònoma de Barcelona (UAB), 08193 Bellaterra (Barcelona), Spain

4 Department of Physical Chemistry, Universitat de Barcelona (UB), C/ Martí i Franquès 1, 08028 Barcelona, Spain

(*) Corresponding author 


\begin{abstract}
The new synthesized thiomacrocyclic ionophore 4-phenyl-4-sulfide-11-(1-oxodecyl)1,7-dithia-11-aza-4-phosphacyclotetradecane has proven to form Langmuir and Langmuir-Blodgett (LB) films. This ionophore shows a large affinity for copper(II) ions. Then, the influence of the subphase composition on the surface pressure-area isotherms has been studied. The LB films have been observed by AFM and the effect of the subphase composition and the deposition surface pressure on the LB films is discussed. AFM image morphology has been correlated to the ionophore molecular structure. Surface pressure-area isotherms and AFM images show that the presence of copper(II) ions has an important role in the film structure.
\end{abstract}

Keywords: Langmuir film, Langmuir-Blodgett film, thiomacrocyclic ionophore, AFM. 


\section{Introduction}

Langmuir-Blodgett (LB) films are nanometric systems of technological and scientific interest, with promising applications in several fields, especially in the field of sensors. Several macrocyclic compounds, as crown ethers, acts as metal ionophores and can be used in the design of chemical sensors [1-4]. The amphiphilic character of some of these macrocyclic compounds opens the possibility to be used in obtaining LB films. Only a few works have been devoted to the study of Langmuir or LB films of macrocyclic compounds, and much less to thiomacrocycles. Lednev and Petty [1,5] studied the amphiphilic dye benzothiazolium styryl containing a crown ether ring, which acts as a silver cation ionophore. Surface pressure measurements in Langmuir monolayers, and ellipsometric and adsorption measurements in LB films were used by the authors to characterise the films and to test the potentiality of the studied compound as a chemical sensor for silver ions. The behaviour of this compound at different subphases was also studied [6]. Gold et al. [2] studied the cation binding of Langmuir films made with poly(4'-vinylbenzo-18-crown-6). Surface pressure measurements using a set of aqueous solutions of alkali metal salts served again to test the potentiality of the ionophore compound to be applied in sensor devices. Winter et al. [7] also reported several crown ether surfactants that form stable monolayers on water, which could be used in cation selective membranes. Matsumura et al. [3, 4] studied, using surface potential measurements, the cation binding of monolayers of octadecyloxymethyl-18-crown-6 and Badis et al. [8] studied the behaviour of several amphiphilic crown ethers and their enantiomeric recognition of amino acids in Langmuir monolayers using surface pressure, BAM images and surface potential measurements. None of the previously cited works have characterised LB films by using atomic force microscopy (AFM). Sergeeva et al. [9] have studied the influence of the counter-anion on the interaction of cations with the benzodithia-18-crown-6 butadienyl dye in monolayers.

The new synthesized thiomacrocyclic ionofore 4-phenyl-4-sulfide-11-(1-oxodecyl)-1,7dithia-11-aza-4-phosphacyclotetradecane (cited in the text as compound IV as described in Figure 1) may also be used for sensing applications, acting as an ionophore of copper ions [10, 11]. Its molecular structure allows forming Langmuir and LB films. In this work we report the physicochemical behaviour of the Langmuir films formed by the 
compound IV, analizyng the surface pressure-area isotherms using pure water and copper ions aqueous solutions as subphases. The characteristics of LB films transferred onto mica from the corresponding monolayers have been observed by AFM and the influence of coppers ions is commented.

\section{Experimental}

\section{Synthesis}

Figure 1 outlines the synthesis of the macrocycle 4-phenyl-4-sulfide-11-(1-oxodecyl)1,7-dithia-11-aza-4-phosphacyclotetradecane (IV). Compounds I and II were prepared as described procedures $[12,13]$. Chemicals used were of the highest purity and all the syntheses were carried out using standard Schlenk techniques under dry nitrogen atmosphere. Solutions of I (4.40 g, $18.1 \mathrm{mmol})$ and II (5.88 g, $18.1 \mathrm{mmol})$ in THF (100 $\mathrm{ml}$ ) were simultaneously added drop wise (rate of $20 \mathrm{ml} / \mathrm{h}$ ) over THF (500 ml) with constant stirring under nitrogen atmosphere. Once addition was completed, the reaction mixture was stirred at room temperature for $24 \mathrm{~h}$ and evaporated to dryness. The resulting white oily material was treated with diethyl ether $(100 \mathrm{ml})$, affording a white solid ( $\mathrm{LiCl})$ which was filtered off and discarded. The ether solution was then evaporated to $5 \mathrm{ml}$ and chromatographied on deoxygenated silica gel. The first fraction eluted with chloroform gives the phosphorous macrocycle III 4-phenyl-11-(1oxodecyl)-1,7-dithia-11-aza-4-phosphacyclotetradecane (1.31 g, $15 \%)$ as a white oil. Sulphuration of III gives the desired macrocycle 4-phenyl-4-sulfide-11-(1-oxodecyl)1,7-dithia-11-aza-4-phosphacyclotetradecane IV. The reaction was carried out by addition of sulphur powder $(0.1 \mathrm{~g}, 2.8 \mathrm{mmol})$ to a solution of III $(0.670 \mathrm{~g}, 1.4 \mathrm{mmol})$ in methanol-toluene $(1: 1$ in volume, $50 \mathrm{ml})$. The resulting suspension was refluxed for $2 \mathrm{~h}$, and after cooling the unreacted sulphur was filtered-off and discarded. The filtrate was concentrated to give crude product which was purified by column chromatography on silica gel. The first fraction eluted with chloroform was collected and evaporated to obtain the macrocycle IV as a white crystalline solid $(0.280 \mathrm{~g}, 40 \%)$. The analyses of the macrocycles III and IV by spectroscopic, ${ }^{1} \mathrm{H}$ NMR and ${ }^{31} \mathrm{P}\left\{{ }^{1} \mathrm{H}\right\} \mathrm{NMR}$, mass spectrometry and analytical methods were found consistent with the predicted structures. 
Macrocycle III: Calc. for $\mathrm{C}_{26} \mathrm{H}_{44} \mathrm{NOPS}_{2}$ : C, 62.92; H, 9.04; N, 2.55; S, 13.38. Found: C, 64.83; H, 9.21; N, 2.91; S, 13.31. MS (EI) m/z [M+H] $]^{+}$482.2(100\%), 483.2(28\%), 484.2(12\%), 485.2(3\%). ${ }^{1} \mathrm{H} \mathrm{NMR}\left(\mathrm{CDCl}_{3}, \delta / \mathrm{ppm}\right), 7.40-7.9(5 \mathrm{H}, \mathrm{m}, \mathrm{Ph}), 3.6-3.2$ (4, m, $-\mathrm{N}-\mathrm{CH}_{2}-$ ), 2.8-2.4 (4H, m, -S-CH$\left.{ }_{2}-\mathrm{CH}_{2}-\mathrm{PPh}\right), 2.60\left(4 \mathrm{H}, \mathrm{m},-\mathrm{N}-\mathrm{CH}_{2}-\mathrm{CH}_{2}-\mathrm{CH}_{2}-\mathrm{S}\right), 2.26$ (2H, pt, - $\left.\mathrm{CH}_{2}-\mathrm{CO}-\right), 2.08$ (4H, m, -S-CH $\left.-\mathrm{CH}_{2}-\mathrm{PPh}\right), 1.80$ (4H, m, - $-\mathrm{N}_{2}-\mathrm{CH}_{2}-\mathrm{CH}_{2}-\mathrm{S}$ ), 1,60-0.84 (17H, m, $\left.\mathrm{CH}_{3}-\left(\mathrm{CH}_{2}\right)_{7^{-}}\right) ;{ }^{31} \mathrm{P}\left\{{ }^{1} \mathrm{H}\right\} \mathrm{NMR}\left(\mathrm{CDCl}_{3}, \delta / \mathrm{ppm}\right),-24.24$.

Macrocycle IV: Calc. for $\mathrm{C}_{26} \mathrm{H}_{44} \mathrm{NOPS}_{3}$ : C, 60.78; H, 8.63; N, 2.73; S, 18.72. Found: C, 60.33; H, 8.61; N, 2.71; S, 18.69. MS (EI) m/z [M+H] $]^{+}, 512.3(7 \%), 513.4$ (55), 514.2(100\%), 515.2(31\%), 516.2(17\%), 517.2(18\%). ${ }^{1} \mathrm{H}$ NMR $\left(\mathrm{CDCl}_{3}, \delta / \mathrm{ppm}\right), 7.81-$ $7.28(5 \mathrm{H}, \mathrm{m}, \mathrm{Ph}), 3.7-3.2\left(4, \mathrm{~m},-\mathrm{N}-\mathrm{CH}_{2^{-}}\right), 3.0-2.2\left(8 \mathrm{H}, \mathrm{m},-\mathrm{S}-\mathrm{CH}_{2}-\mathrm{CH}_{2}-\mathrm{PPh}\right), 2.65$ (4H,m, $\left.-\mathrm{N}-\mathrm{CH}_{2}-\mathrm{CH}_{2}-\mathrm{CH}_{2}-\mathrm{S}\right), 2.30$ (2H, pt, $\left.-\mathrm{CH}_{2}-\mathrm{CO}-\right), 1.97$ (4H, m, - $-\mathrm{CH}_{2}-\mathrm{CH}_{2}-\mathrm{CH}_{2}-$ $\mathrm{S}), 1,65-0.84\left(17 \mathrm{H}, \mathrm{m}, \mathrm{CH}_{3}-\left(\mathrm{CH}_{2}\right)_{7^{-}}\right) ;{ }^{31} \mathrm{P}\left\{{ }^{1} \mathrm{H}\right\} \mathrm{NMR}\left(\mathrm{CDCl}_{3}, \delta / \mathrm{ppm}\right), 46.29$.

\section{Isotherms, LB and AFM}

Surface pressure-area isotherms were obtained in a NIMA 1232D1D2 LangmuirBlodgett trough ( $a r e a=1200 \mathrm{~cm}^{2}$ ) placed on an isolation platform. $50 \mu$ l of a solution of compound IV in chloroform, at a concentration of $1 \mathrm{mg} / \mathrm{ml}(1.9 \mathrm{mM})$, was spread over the corresponding subphase, and $15 \mathrm{~min}$ were lag before measurements in order to permit evaporation of the solvent. The compression speed was of $50 \mathrm{~cm}^{2} \mathrm{~min}^{-1}$, that is $2.5 \mathrm{~cm} \min ^{-1}$ or $8.5 \AA^{2}$ molecule $\mathrm{e}^{-1} \mathrm{~min}^{-1}$. The surface pressure was measured using the Wilhelmy method incorporated to the NIMA trough and a paper sheet. Pure water, Millipore MilliQ grade (resistivity of $18 \mathrm{M} \Omega \mathrm{cm}$ ), and solutions of copper(II) nitrate (from analytical reagent $\mathrm{Cu}\left(\mathrm{NO}_{3}\right)_{2}$ and MilliQ water) were used as subphases.

The LB films were transferred at constant pressure onto a fresh cleaved atomically flat mica surface. The transfer was done from the corresponding films on water, using a NIMA $1232 \mathrm{D} 1$ dipper, at several surface pressures and at $21^{\circ} \mathrm{C}$. The substrates were sheets of $1 \mathrm{~cm} \times 1 \mathrm{~cm}$ cut from reed mica. LB films were obtained following a $\mathrm{Z}$ deposition, that is, the sheet of mica is first introduced in the subphase, and once the Langmuir film is formed, the mica sheet is pulled up, with a extraction speed of 10 $\mathrm{mm} / \mathrm{min}$. The obtained transfer ratios were close to $100 \%$. AFM images were obtained 
in a Nanoscope IIIA (Digital Instruments, CA) using contact and tapping modes. Tapping mode images were obtained using silicon microfabricated cantilevers with a constant force of $40 \mathrm{~N} / \mathrm{m}$ and $300 \mathrm{kHz}$ frequency, meanwhile contact mode images were obtained with a silicon nitride cantilever, nominal force constant of $0.16 \mathrm{~N} / \mathrm{m}$.

\section{Results and discussion}

\section{Surface pressure-area isotherms}

Figure 2 shows the surface pressure-area isotherms for a spread film of the thiomacrocyclic compound IV on the two mentioned subphases, at $21^{\circ} \mathrm{C}$ : curve a) was obtained using water as subphase, and curve b) using a $0.05 \mathrm{M}$ aqueous solution concentration of copper(II) ions. The comparison of both isotherms shows the notable influence of copper ions, fact that reinforce the ligand character of the thiomacrocyclic compound with the copper cation. Curve b) reaches a collapse at the surface pressure of $28.5 \mathrm{mN} / \mathrm{m}$ but curve a) develops a quasi plateau far bellow the collapse pressure of curve b). Otherwise, the quasi plateau extends till an area per molecule which have no sense. Cyclic isotherms were performed to get more information and figure 3 shows the corresponding cyclic isotherms to curves a) and b) of Figure 2. Meanwhile, curve a) (water subphase) shows a quasi reversible behaviour, even when high area compressions were reached, that is not the case for curve b) (subphase of copper ions), where an important hysteresis happens. If the cyclic isotherm for curve b) is limited to a surface pressure value below the collapse pressure (inset of Figure 3), a reversible behaviour is registered. Then, the state reached at the plateau for curves a) and b) is quite different, and only curve b) reaches a real or catastrophic collapse. This point will be discussed below in connection with the AFM images. The effect of the copper ion concentration in the subphase is shown in Figure 4: An increase in copper ion concentration produces that the film will attain higher values of surface pressure.

The compressibility coefficient $\beta=-1 / \mathrm{A}(\delta \mathrm{A} / \delta \pi)_{\mathrm{T}}$ for the ascending part of isotherm a) in Figure 2 gives a value of $\approx 2510^{-3} \mathrm{~m} / \mathrm{mN}$, this value corresponding to a liquid expanded 
(LE) state [14]. The ascendant part of curve b) in Figure 2 shows an inflexion, at $\pi \approx 17$ $18 \mathrm{mN} / \mathrm{m}$, and the compressibility coefficient $\beta$ is about $2210^{-3} \mathrm{~m} / \mathrm{mN}$ in the range of low pressure values and about $4210^{-3} \mathrm{~m} / \mathrm{mN}$ in the range of high pressure values. These values correspond also to a LE state, so the inflexion does not indicate a change from LE state to LC, but some kind of rearrangement of the molecules in the monolayer. This point will be discussed below.

\section{AFM of LB films}

Figures 5-7 show AFM images of the LB films transferred onto mica from the subphase containing copper(II) ions. AFM images of LB films deposited at a value $10 \mathrm{mN} / \mathrm{m}$ of surface pressure always show big homogeneous areas only perturbed by small islands that protruded from them (Figure 5a). Higher resolution images in the homogeneous areas show the nanometric texture of the film (Figures $5 \mathrm{~b}$ and $5 \mathrm{c}$ ). In the images it is possible to measure the film height due to the presence of nanometric voids, which results of $1.0 \mathrm{~nm}$ (Figure 5c). The monolayer height has also been determined by scratching the film with the AFM tip, and again a value of $1 \mathrm{~nm}$ was measured. This value is in accordance with the value deduced from the molecular structure of compound IV considering that the ring is placed planar to the surface with the alkyl chain and the benzene ring oriented to the air phase (see discussion section). We can conclude that a monolayer is covering the mica surface in these homogeneous areas. At a high surface pressure value of $25 \mathrm{mN} / \mathrm{m}$, the film is more compact and AFM images do not show the voids (Figure 6). Figure 7 is a scanned zoom of Figure 5a showing the structure of an island. Profile section indicates that the height of the island is around 2 $\mathrm{nm}$, and that a higher protrusion arises in the centre, also measuring around $2 \mathrm{~nm}$. These heights are multiples of the monolayer height.

When the value of the deposition surface pressure is increased, the formation of bigger and taller islands is observed. For comparison, Figure 8a corresponds to the copper solution subphase at a surface pressure of $25 \mathrm{mN} / \mathrm{m}$ and Figure $8 \mathrm{~b}$ corresponds to the water subphase and at a deposition surface pressure in the plateau of the isotherm. In the case of water subphase the number of island per unit area is much higher than in the case of copper ions solution subphase, then showing a clear influence of the subphase. 
These islands have also been observed in the Langmuir film at the water-air interphase using a home-made prototype of Brewster angle microscope, still not fully operative

\section{Discussion}

The study of the $\pi$-A isotherms and AFM images suggests the formation of a monomolecular film with few islands on it, which formation depends on the subphase composition and the applied surface pressure. The monolayer height of $1 \mathrm{~nm}$ measured by contact mode AFM agrees with the vertical dimension of the molecule as given in Figure 9, taking into account that the hydrocarbon tail can adopt less extended conformations and that the cantilever realise a force on the top of these tails. LB films transferred to mica remains stable for several days, and even we do not have a direct correlation with the structure of the Langmuir film, it looks that a monolayer is also present in the air-water interphase.

The formation of monolayer films has been reported previously for macrocyclic compounds $[1,6-9,15,16]$ even though, depending on the subphase, the substrate and the specific structure of the compound, multilayer formation has also been observed [17-19]. Armstrong et al. [17-19] proposed a bilayer for cooper phthalocyanine derivatives, but observed a clear transition in the isotherm that is not our case. Jiao and Liu [20] reported the formation of monolayers or multilayers in naphthylidenecontaining Schiff base derivatives, depending on the compound structure. Using AFM, these authors also observed the formation of nanofibers for some of these compounds, depending on the substituted functional groups. In our case, the particular shape of the isotherms and the structure of the films observed by AFM, are a consequence of the particular molecular structure of the thiomacrocyclic compound. Small surface pressure values were also reported for macrocyclic compounds, as amphiphilic crown ethers [8].

As commented above, the number and dimension of the islands presents in the film increase with the surface pressure. In the water subphase, the number of islands per unit area increases notably in respect to the copper ions subphase. Concluding, the existence of an interaction between copper ions and the ligand molecules, more specifically with the macrocycle part, allows the films on a copper ions solution subphase to reach higher 
surface pressures, and consequently higher compactness (curve b) in Figure 2). The value of $40 \mathrm{~A}^{2} /$ molecule at which collapse occurs in curve b) is close to the geometrically projected molecular area [21] in Figure 9. The molecular dimensions were obtained using Chem3D Ultra from Cambridgesoft. Chem3D obtains data for the MM2 calculations from tweaked version of the MM282 parameter set. Values in between 40 and $100 \mathrm{~A}^{2} /$ molecule have been reported for different crown ether [2, 3, 7 , $8,13,22,23]$. Figure 10 shows a tentative correspondence between the different parts of the isotherm and the conformations that the thiomacrocyclic molecules can adopt in the film. At null pressure, the molecule present an extended conformation (I, in figure 10), corresponding to a gas phase, with the hydrocarbon chain free to move to a position lying almost parallel to the surface, until a maximum disposed area per molecule of around $100 \AA^{2}$. Further compression leads to a forced rearrangement of the hydrocarbon chain more perpendicular to the surface (II in Figure 10), corresponding to a LE phase, until an area per molecule of around $65 \AA^{2}$. In order to favour lateral interactions, it seems plausible a tail-to-tail disposition. Higher compressions may produce a disposition of the benzene ring more perpendicular to the surface (III in Figure 10), until to reach an area per molecule of $40 \AA^{2}$. After that, when area compression has reached the molecular area, collapse occurs because no further molecular structure compression can be done.

On a pure water subphase, the isotherm shows a quasi plateau. In several works [24-27] the formation of a trilayer by the roll-over mechanism has been proposed, always linked with the development of a plateau in the isotherm. In our case, AFM images do not indicate that a homogeneous trilayer is formed, but only random distributed islands of several $\mathrm{nm}$ height are observed in the quasi plateau region. Also, the reversible compression till very small areas per molecule not having physical sense reinforces the idea that a trilayer formation mechanism does not happen. A different mechanism is operating in our case and the plateau formation does not correspond to a phase transition as usually defined in Langmuir films.

In the case of planar macrocyclic compounds with $\pi$ conjugated systems, as phthalocyanines, the stacking face-to-face of the planar rings is usual, favoured by the $\pi-\pi$ interactions. An edge-on arrangement leads to the formation of rodlike entities, especially at higher compressions $[15,16]$. These arrangements are dependents on the 
subphase and the particular molecular compound structure [28]. In our case the thiomacrocyclic ring does not have a $\pi$ conjugated systems and is not totally planar, then regular stacking is not favoured in the absence of the $\pi-\pi$ interactions, and the formation of rodlike aggregates has not been observed by AFM.

In the case of the water subphase, the molecules of compound IV are not strongly attached to the subphase because the macrocyclic ionophore molecules do not have a well defined hydrophilic head group. This fact and the presence of the macrocycle will favour the slip off of the molecules from the monolayer film to island structures, when the film is compressed (IV in Figure 10) most probably happening at defect positions in the film. At low compressions, the island height is only one or a few nanometers and some of the islands present also a small protrusion in the centre, as given in Figure 7. Compound IV molecules can then be locally pushed up forming an aggregate on the surface that lately spreads forming a circular island. At high compressions, the island height is of several nanometers, around 14-16 nm. AFM images in Figure 8 show clearly this fact. These bigger islands correspond to aggregates where the cohesion forces between molecules prevent its spread. The isotherm plateau reached at low surface pressures in the water subphase does not correspond to a real collapse, but to a stage where a lot of island structures are formed and coexist with the monolayer. Figure 3 shows the reversible character of this phenomenon. On the contrary, in the copper subphase, copper ions bind to the macrocycle from the solution side and difficult the formation of these island structures. Consequently, even a much higher surface pressure is reached, the number of formed islands is less. $\mathrm{So}, \mathrm{Cu}$ (II) ions play an important role in the morphology of the monolayer. The slip off of the molecules from the monolayer film to form island structures, also explains that the calculated compressibility coefficient presents higher values at higher compressions.

The dependence on copper ion concentration in the subphase as observed in the isotherms of Figure 4, points to the existence of an equilibrium distribution of $\mathrm{Cu}$ (II) ions between the solution subphase as solvated ions an the Langmuir film as complex $\mathrm{Cu}$ (II)-macrocycle. Figure 11 shows the values of surface pressure reached in the isotherms, at an area per molecule close to the collapse, versus the copper ion concentration in the subphase. At a fixed area per molecule close to the collapse value, an increase of the copper concentration in solution produces an increase in the surface 
pressure, till saturation for concentration values higher than $0.03 \mathrm{M}$. Thus, we can establish that at $\left[\mathrm{Cu}^{2+}\right]>0.03 \mathrm{M}$, the whole thiomacrocycle molecules in the Langmuir

film are in its complex form with copper ions, $\Gamma_{\text {total }} \approx \Gamma_{\text {complex }}$, but at $\left[\mathrm{Cu}^{2+}\right]<0.03$ a certain ratio of not bound macrocyclic molecules, $\Gamma_{\text {free, }}$ still remains.

New experiments are planned to observe Langmuir films by BAM and to study molecular orientations in the Langmuir and LB films.

\section{Summary}

The formation of Langmuir and Langmuir-Blodgett films of a new thiomacrocyclic compound has been studied. The presence of copper(II) ions in the subphase has a crucial effect on the film structure, due to the affinity between copper ions and the thiomacrocycle. AFM images reveal the morphological characteristics of the LangmuirBlodgett films at different values of the deposition surface pressure and the influence of the subphase.

\section{Acknowledgements}

This work has been supported by Generalitat de Catalunya trough project 2002PIRA 00167, and by MCYT trough projects TIC2002-04280-C03-01 and CTQ2004-08046C02 and -01. One of the authors, J. T-B., thanks to the UPC for granting him a sabbatical year.

\section{References}

[1] I.K. Lednev and M.C. Petty, J. Phys. Chem., 98 (1994) 9601.

[2] J.M. Gold, D.M. Teegarden, K.M. McGrane, D.J. Luca, P.A. Falcigno, C.C. Chen and T.W. Smith, J. Am. Chem. Soc., 108 (1986) 5827.

[3] H. Matsumura, K. Furusawa, S. Inokuma and T. Kuwamura, Chem. Lett., (1986) 453. 
[4] H. Matsumura, T. Watanabe, K. Furusawa, S. Inokuma and T. Kuwamura, Bull. Chem. Soc. Jpn., 60 (1987) 2747.

[5] I.K. Lednev and M.C. Petty, Langmuir, 10 (1994) 4190.

[6] I.K. Lednev and M.C. Petty, Langmuir, 10 (1994) 4185.

[7] H-J. Winter and G. Manecke, Makromol. Chem., 186 (1985) 1979.

[8] M. Badis, I. Tomaszkiewicz, J-P. Joly and E. Rogalska, Langmuir 20 (2004) 6259.

[9] T.I. Sergeeva, S.P. Gromov, A.I. Vedernikov, M.S. Kapichnikova, M.V. Alfimov, V-T. Lieu, D. Möbius, M.S. Tsarkova and S. Yu. Zaitsev, Colloids \& Surf. A 255 (2005) 201.

[10] I.A. Marques de Oliveira, M. Pla-Roca, L. Escriche, J. Casabó, N. Zine, J.

Bausells, F. Bessueille, J. Samitier and A. Errachid, IEEE Sensors 2004, The $3^{\text {rd }}$ IEEE Conference on Sensors, Vienna (Austria), 2004.

[11] I.A. Marques de Oliveira, M. Pla-Roca, L. Escriche, J. Casabó, N. Zine, J. Bausells, J. Samitier and A. Errachid, Mat. Sci. Eng. C, accepted, in press.

[12] I.A. Marques de Oliveira, J. Torrent-Burgués, M. Pla, N. Zine, J. Bausells, Ll. Escriche, J. Casabó, A. Errachid and J. Samitier, Analytical Letters, accepted, in press.

[13] J.A. Muñoz, L. Escriche, J. Casabo, C. Perez-Jimenez, R. Kivekas and R. Sillanpaa, Inorg. Chim. Acta, 257(1) (1997) 99.

[14] S. Hauptmann and A.W. Poege, Pharmazie, 28(8) (1973) 520,

[15] L. Hedvati, A. Nudelman, E. Falb, B. Kraiz, R. Zhuk and M. Sprecher, Eur. J. Med. Chem., 37(7) (2002) 607.

[16] M.C. Petty, Langmuir-Blodgett films. An introduction, Cambridge University Press, Cambridge, 1996.

[17] H-Q. Xiang, K. Tanaka, A. Takahara and T. Kajiyama, Lang 18 (2002) 2223.

[18] S. O'Flaherty, L. Wiegart, O. Konovalov, B. Struth, Lang 21 (2005) 11161.

[19] P.E. Smolenyak, E.J. Osburn, S-Y. Chen, L-K. Chau, D.F. O’Brien, N.R. Armstrong, Lang 13 (1997) 6568;

[20] P. Smolenyak, R. Peterson, K. Nebesny, M. Törker, D.F. O’Brien, N.R. Armstrong, J. Am. Chem. Soc. 121 (1999) 8628.

[21] C.L. Donley, W. Xia, B.A. Minch, R.A.P. Zangmeister, A.S. Drager, K. Nebesny, D.F.O’Brien, N.R. Armstrong, Lang 19 (2003) 6512.

[22] W. Xia, B.A. Minch, M.D. Carducci, N.R. Armstrong, Lang 20 (2004) 7998.

[23] T. Jiao, M. Liu, J. Colloid Interface Sci, in press

[24] M. Pla, Ph Thesis, Universitat Autònoma de Barcelona (UAB), 2004. 
[25] I.K. Lednev and M.C. Petty, Adv. Mater. Opt. Electr., 4 (1994) 225.

[26] A. Ulman, An Introduction to Ultrathin Organic Films, Academic Press, Boston, 1990.

[27] M.C. Friedenberg, G.G. Fuller, C.W. Frank and C.R. Robertson, Lang. 10 (1994) 1251.

[28] M.N.G. de Mul and J. Adin Mann, Jr., Lang. 10 (1994) 2311.

[29] J.A. Schröter, R. Plehnert, C. Tschierske, S. Katholy, D. Janietz, F. Penacorada and L. Brehner, Lang. 13 (1997) 796.

[30] M. Pérez-Morales, J.M. Pedrosa, M.T. Martín-Romero, D. Möbius and L. Camacho, J. Phys. Chem. B, 108 (2004) 4457.

[31] S. Fouriaux, F. Armand, O. Araspin, A. Ruaudel-Teixier, E.M. Maya, P. Vazquez, T. Torres, J. Phys. Chem. 100 (1996) 16984. 


\section{Figures}

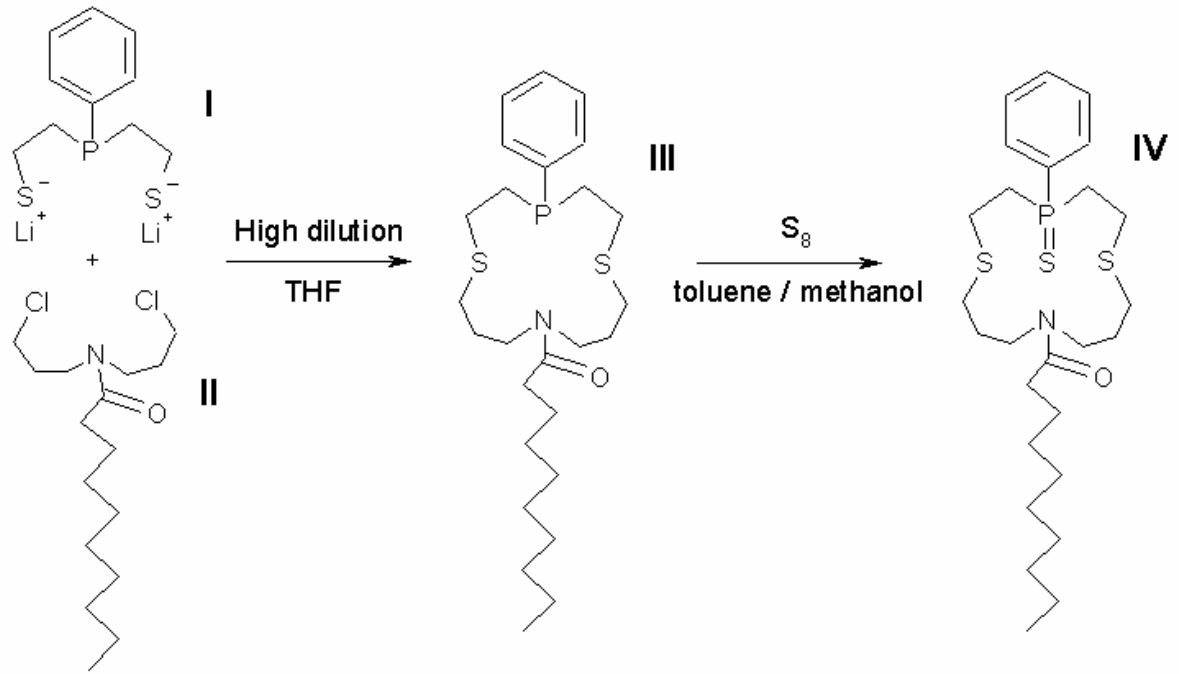

Figure 1. Synthesis pathway

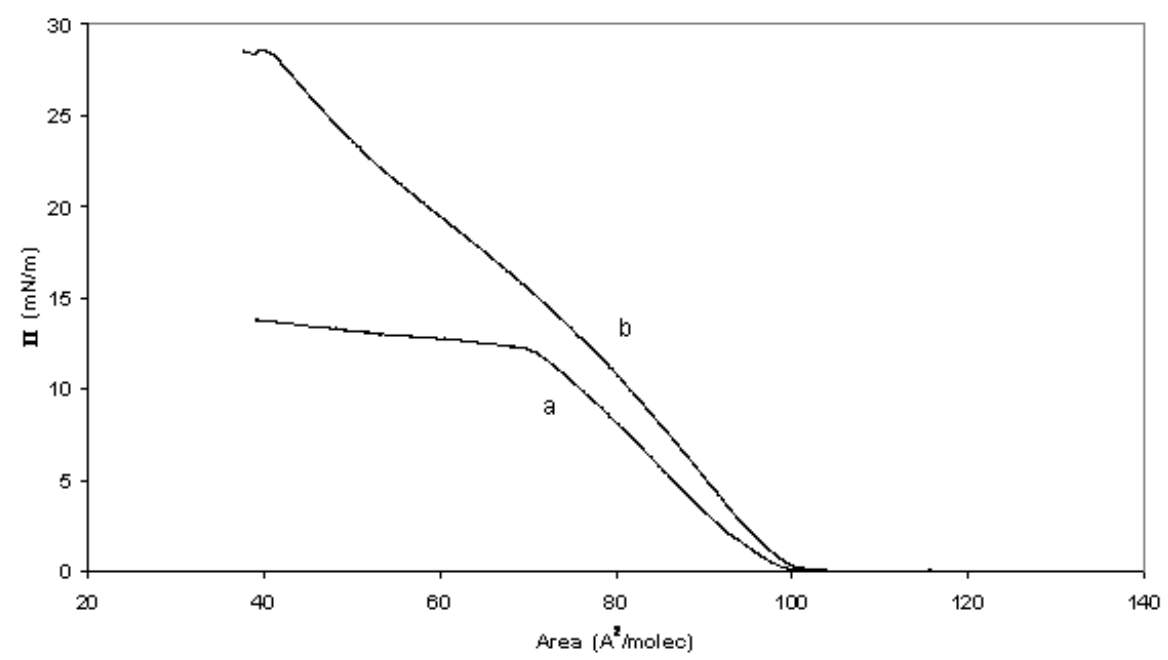

Figure 2. Surface pressure-area isotherms for Langmuir films of compound IV on different subphases: a) water, b) aqueous solution $0.05 \mathrm{M}$ in copper(II) ions. 


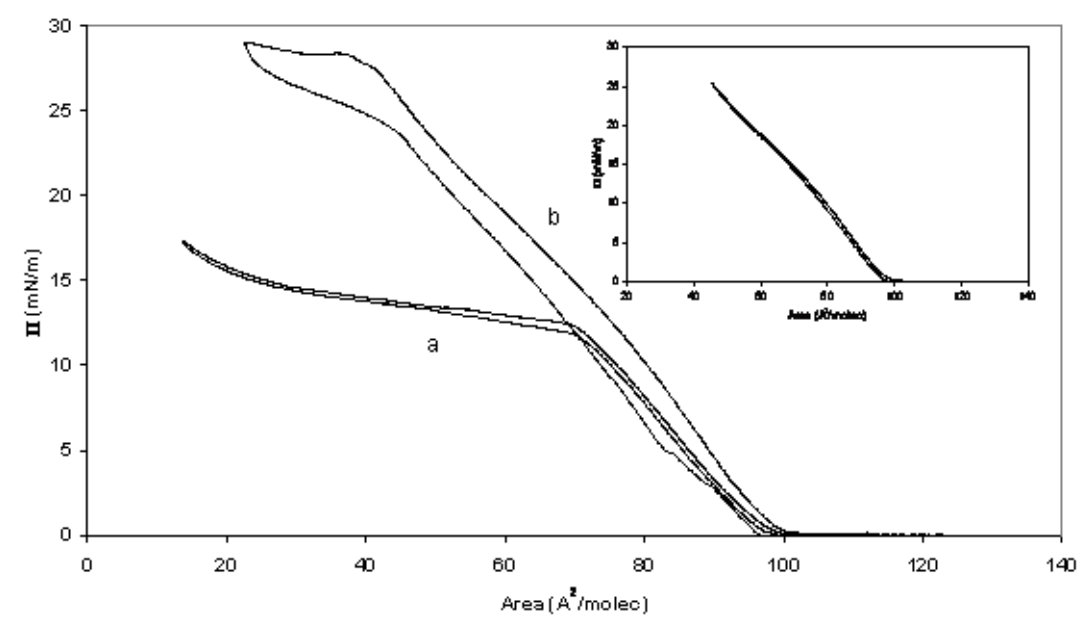

Figure 3. Hysteresis in a compression-expansion cycle for a spread film of compound IV on two subphases: a) water, b) aqueous solution $0.05 \mathrm{M}$ in copper(II) ions. Inset: Hysteresis cycle for b) when the barrier scan was reversed at a surface pressure value below the collapse.

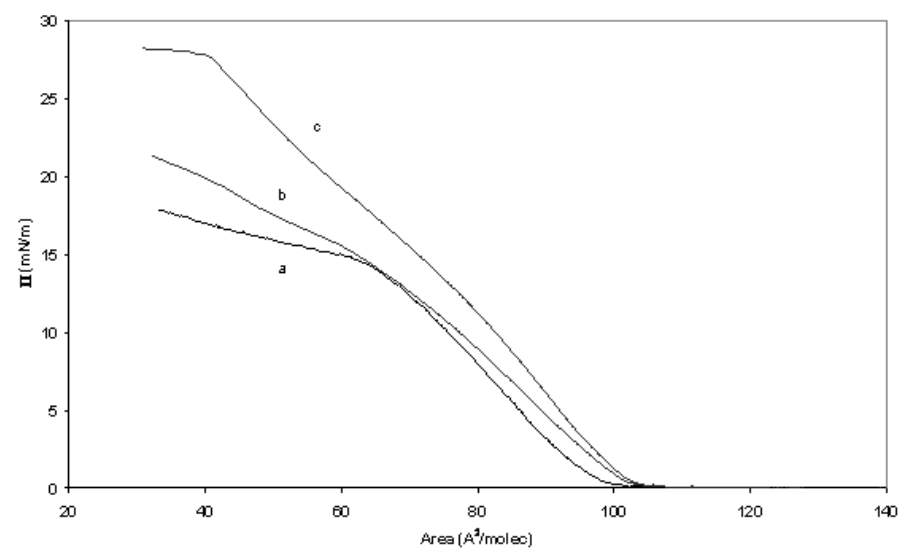

Figure 4. Surface pressure-area isotherms for Langmuir films of compound IV on different aqueous copper subphases: a) $0.001 \mathrm{M}$ in $\mathrm{Cu}(\mathrm{II})$, b) $0.01 \mathrm{M}$ in $\mathrm{Cu}(\mathrm{II})$, c) 0.05 $\mathrm{M}$ in $\mathrm{Cu}(\mathrm{II})$. 

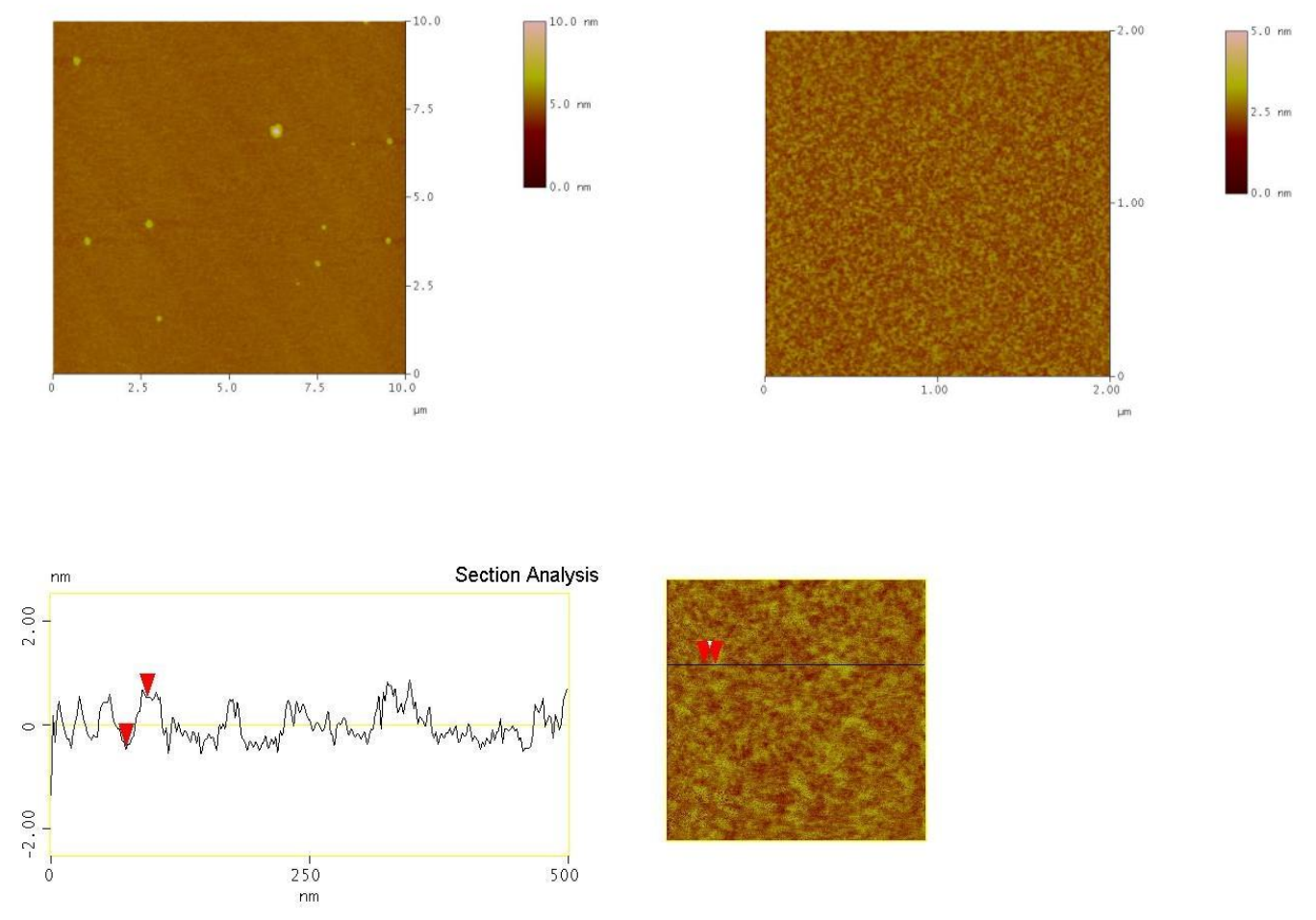

Figure 5. AFM images of a LB deposited at $10 \mathrm{mN} / \mathrm{m}$ from a $0.05 \mathrm{M}$ copper ions subphase: a) tapping mode, area 10x10 $\mu \mathrm{m}$, b) contact mode, $2 \times 2 \mu \mathrm{m}$, c) section of an area $0.5 \times 0.5 \mu \mathrm{m}$.

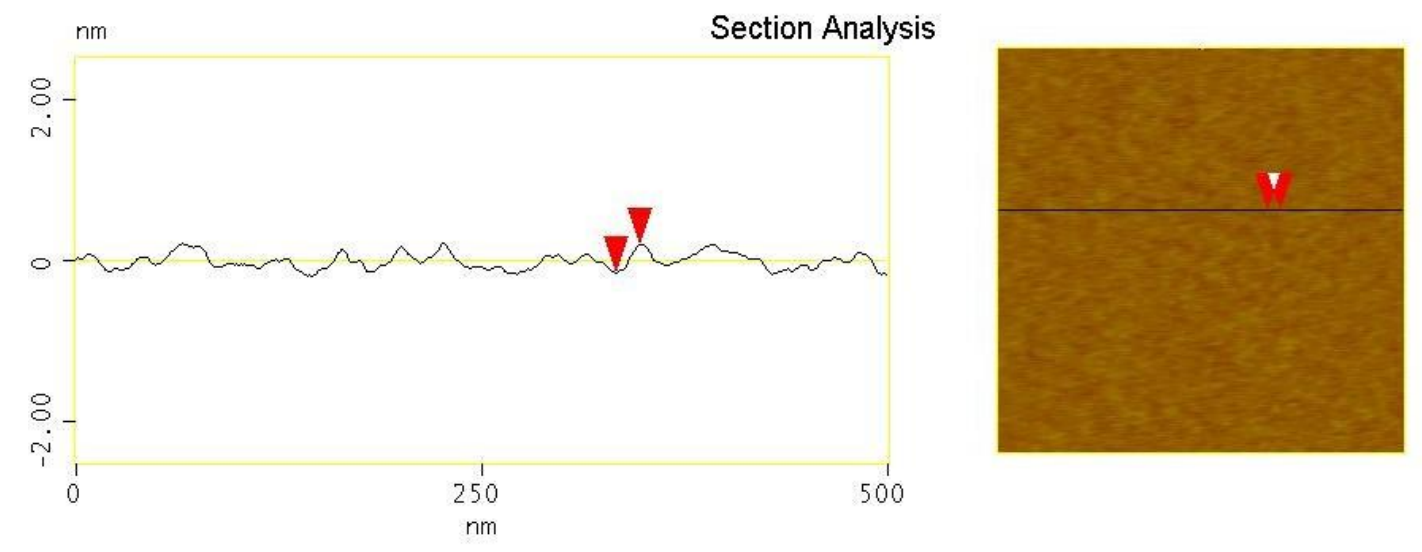

Figure 6. AFM image in contact mode, and section, of a LB deposited at $25 \mathrm{mN} / \mathrm{m}$ from a $0.05 \mathrm{M}$ copper ions subphase; area $0.5 \times 0.5 \mu \mathrm{m}$. 

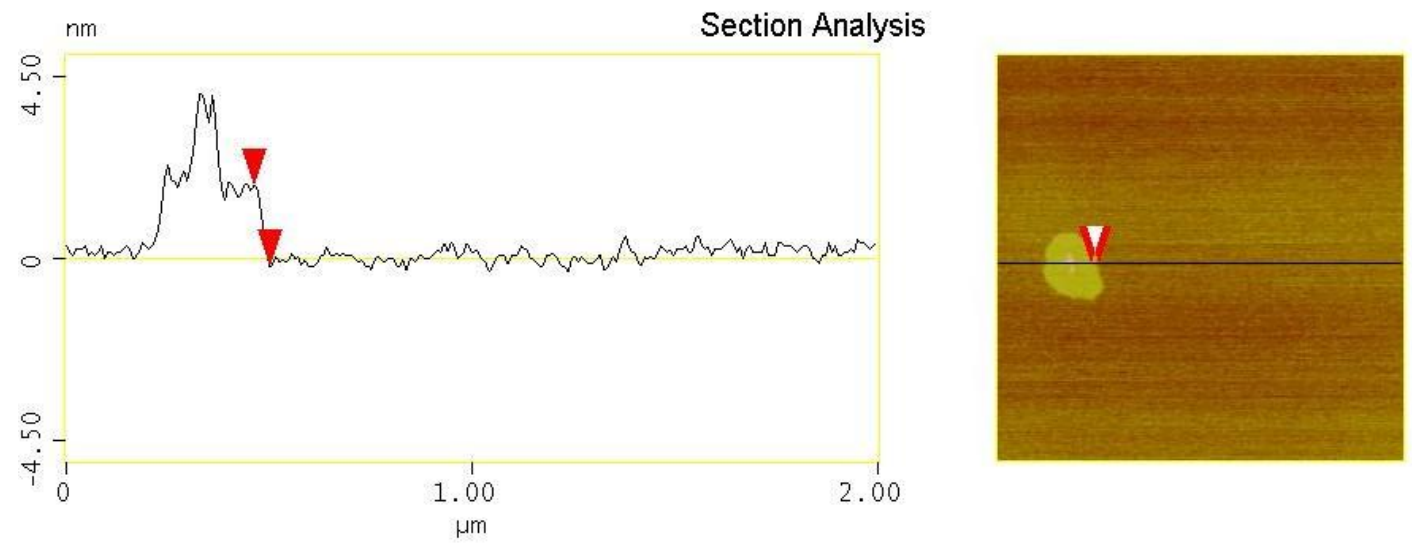

Figure 7. AFM image and section of an island of figure 5a; area $2 \times 2 \mu \mathrm{m}$.
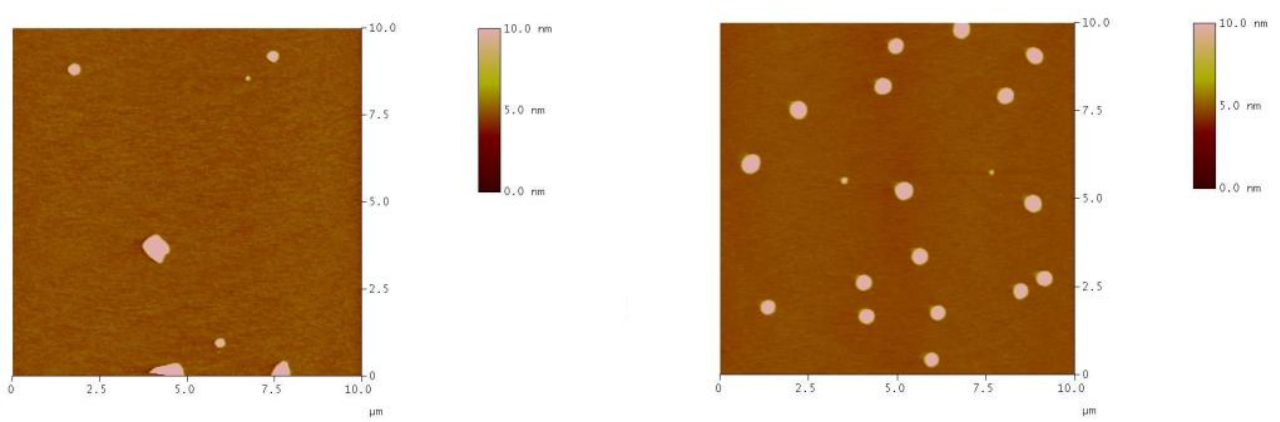

Figure 8. AFM images, in tapping mode, of LB films from a subphase with: a) copper ions solution at a surface pressure of $25 \mathrm{mN} / \mathrm{m}$, b) water at a surface pressure of 13 $\mathrm{mN} / \mathrm{m}$. Scan area $10 \times 10 \mu \mathrm{m}$. 


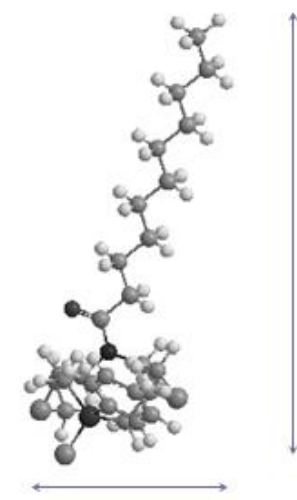

$5.3 \mathrm{~A}$

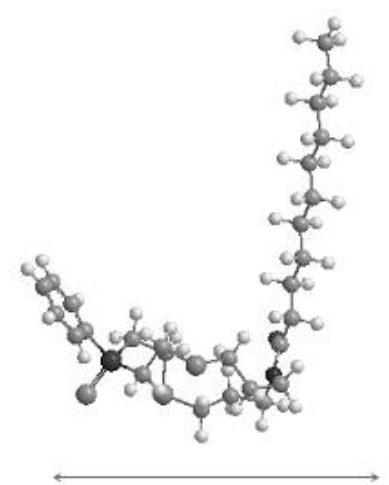

$11.4 \mathrm{~A}$

Figure 9. Molecular dimensions of the compound IV.

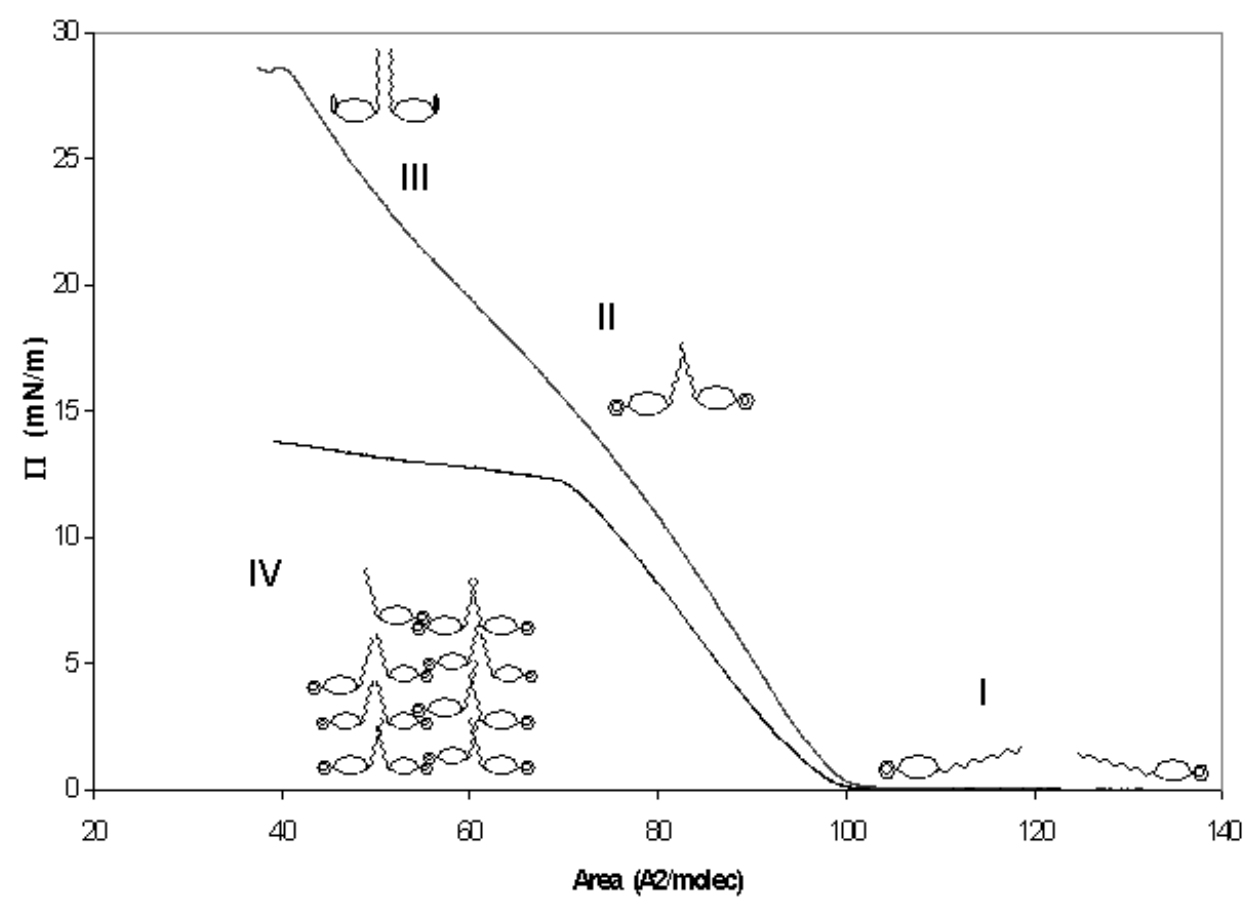

Figure 10. Cartoon modelling in a simple way molecular orientation (I-III) and island formation (IV) in Langmuir films for compound IV. 


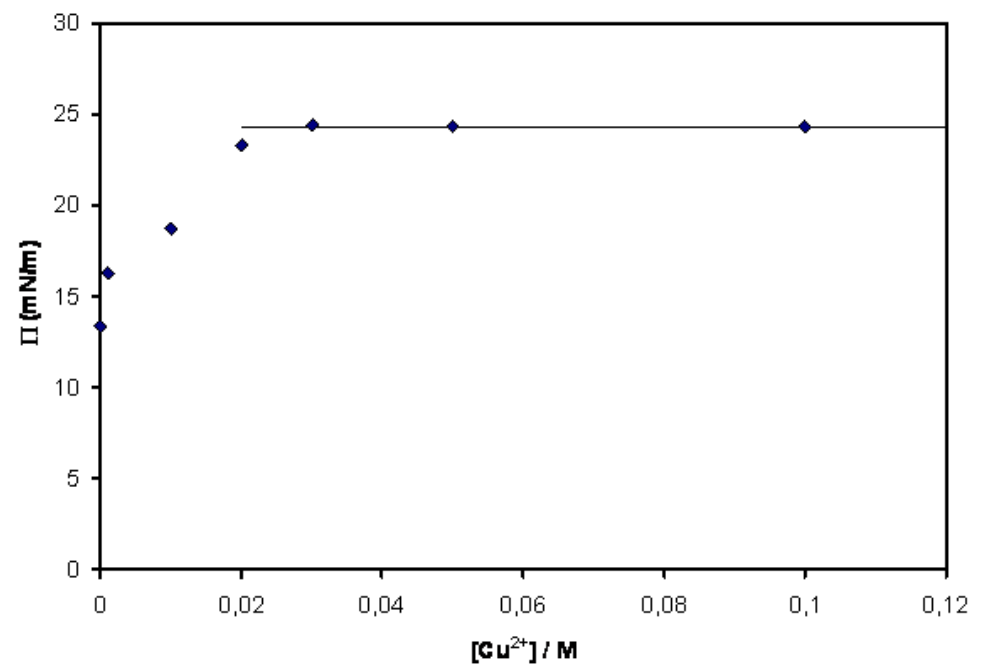

Figure 11. Surface pressure versus copper(II) ion concentration in the subphase, at a fixed area per molecule of $45 \AA^{2} /$ molec. 